\title{
Visual Attention in Autism Families: 'Unaffected' Sibs Share Atypical Frontal Activation
}

Running title: Visual Attention in Autism Families

Matthew K. Belmonte ${ }^{\mathrm{a}, \mathrm{c}, *}$ Marie Gomot ${ }^{\mathrm{b}, \mathrm{c}}$ Simon Baron-Cohen ${ }^{c}$

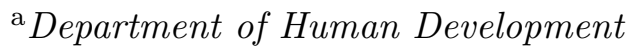
Cornell University, Ithaca, New York

${ }^{\mathrm{b}}$ INSERM U619, Université François-Rabelais, CHRU de Tours, France

${ }^{\mathrm{c}}$ Autism Research Centre

Department of Psychiatry

University of Cambridge, UK

Corresponding author: Matthew K. Belmonte

Department of Human Development

Martha Van Rensselaer Hall

Cornell University

Ithaca, NY 14853-4401

belmonte@mit.edu

voice: +1.617 .715 .2049$

fax: +1.607 .255 .9856$

Conflict of interest statement: No conflicts declared.

239 words in abstract

7530 words in text

3 tables

6 figures 


\section{Abstract}

Background: In addition to their more clinically evident abnormalities of social cognition, people with autism spectrum conditions (ASC) manifest perturbations of attention and sensory perception which may offer insights into the underlying neural abnormalities. Similar autistic traits in ASC relatives without a diagnosis suggest a continuity between clinically affected and unaffected family members. Methods: We applied fMRI in the context of a non-social task of visual attention in order to determine whether this continuity persists at the level of brain physiology. Results: Both boys with ASC and clinically unaffected brothers of people with ASC were impaired at a visual divided-attention task demanding conjunction of attributes from rapidly and simultaneously presented, spatially disjoint stimuli and suppression of spatially intervening distractors. In addition, both groups in comparison to controls manifested atypical fronto-cerebellar activation as a function of distractor congruence, and the degree of this frontal atypicality correlated with psychometric measures of autistic traits in ASC and sibs. Despite these resemblances between the ASC and sib groups, an exploratory, hypothesis-generating analysis of correlations across brain regions revealed a decrease in overall functional correlation only in the ASC group and not in the sibs. Conclusions: These results establish a neurophysiological correlate of familial susceptibility to ASC, and suggest that whilst abnormal time courses of frontal activation may reflect processes permissive of autistic brain development, abnormal patterns of functional correlation across a wider array of brain regions may relate more closely to autism's determinants.

Key words: autism, broader autism phenotype, visual spatial attention, functional MRI, frontal cortex, cerebellum, intraparietal sulcus, cingulum, functional connectivity 


\section{Introduction}

Autism is a behaviourally defined condition whose diagnosis depends on a 'triad' of deficits comprising impaired social interaction, impaired communication, and restricted interests and repetitive behaviours. Symptoms in these domains vary greatly across individuals, making autism the extreme of a spectrum or continuum of abnormalities whose milder end is not necessarily distinct from variation in the general population (Constantino et al., 2003). This continuous variation often manifests in autism families as the Broader Autism Phenotype, a subclinical pattern of cognitive and behavioural traits involving mild social and communicative deficits (Piven et al., 1997; Baron-Cohen \& Hammer, 1997) and associated with a cognitive style biased towards local details (Happé et al., 2001; Baron-Cohen \& Hammer, 1997). Despite many behavioural and psychometric results relating ASC and the Broader Autism Phenotype (reviewed in Belmonte et al. (2004b)), comparatively little attention has been given to physiological investigations of these familial patterns.

Three studies have uncovered tantalising abnormalities in the neural bases of social perception in first-degree relatives: The brains of parents of autistic children seem less specialised for face perception, lacking the normal rightlateralised augmentation of the N170 event-related potential to faces, and lacking the normal correlation between N170 amplitude and face expertise

\footnotetext{
* Department of Human Development Martha Van Rensselaer Hall

Cornell University

Ithaca, NY 14853-4401

voice: +1.617 .715 .2049$

fax: +1.607 .255 .9856$

Email addresses: belmonte@mit.edu (Matthew K. Belmonte),

m.gomot@chu-tours.fr (Marie Gomot), sb205@cam.ac.uk (Simon Baron-Cohen). URL: http://www.mit.edu/ belmonte/ (Matthew K. Belmonte).
} 
(Dawson et al., 2005). Parents also hyperactivate frontal cortex during the Reading the Mind in the Eyes Test (Baron-Cohen et al., 2006b) - perhaps as a compensatory strategy for this perceptual difference. Likewise, clinically unaffected sibs of children with autism have abnormally small amygdalae, gaze at eyes abnormally infrequently, and activate the right fusiform face area less than controls do (Dalton et al., 2007). Dawson et al. (2005, p. 693) have suggested that such social perceptual impairments may develop from a convergence of abnormalities of social motivation and reward on the one hand, and fundamental abnormalities of perception on the other. Indeed, early-developing, non-social perceptual abnormalities, such as sensory-seeking behaviours and long latency to disengage attention, combine with early social abnormalities in predicting later diagnosis of autism (Zwaigenbaum et al., 2005). It thus seems warranted to examine the neural bases of non-social as well as social traits as potential markers of familial autism susceptibility.

One possible unifying theme between social and non-social domains is the notion of selective deficits in 'complex' processing. Within the domain of visual perception, autistic impairments at detecting coherent motion (Milne et al., 2005) and second-order contrast (Bertone et al., 2005) have led to the hypothesis that first-order perceptual computations may take precedence over more complex perceptual processing (Bertone et al., 2003), analogously to the autistic profile of precedence of local over complex information processing in higher-order cognitive domains (Minshew et al., 1997). In both of these cases, the cognitive and the perceptual, 'complexity' is somewhat domain-specific in its particulars (Minshew et al. 1997, p. 312) but can be understood in general as a requirement for rapid and integrative processing of information from multiple inputs (Minshew et al. 1997, p. 313). 
One method of quantifying this integrative demands draws on the cognitive theory of relational complexity. Simply put, the relational complexity of a cognitive or perceptual operation is the number of interacting variables (the integrative aspect of Minshew's sense of complexity) that must be represented simultaneously (the rapid aspect) in the process of performing the operation (Halford et al., 1998). For instance, evaluating second-order (textural or multiplicative) contrast requires the integration of local luminance contrasts over an interval of spatial positions and thus depends on the luminance $\times$ position interaction, whereas first-order (luminance or additive) contrast can be assayed from a single local contrast signal. Although the theory of relational complexity has found most of its application at more abstract, cognitive levels of processing (e.g. in characterising the complexity of false-belief tasks (Andrews et al., 2003)), as a computational description it can be applied equally well in the domain of perceptual integration. This notion of complexity-specific deficits across domains relates well to ideas of atypical neural connectivity in autism (Brock et al., 2002; Belmonte et al., 2004a; Just et al., 2004; Courchesne and Pierce, 2005).

A recent elaboration of the Enhanced Perceptual Function theory of autism (Mottron et al., 2006) cites abnormally high salience at high levels of specialisation for the central, high-magnification portion of the visual field (consistent with behavioural and physiological findings of a narrow, 'spotlight' distribution of spatial attention in autism (Townsend et al., 1994)), and at low levels of the hierarchy of visual processing abstraction (consistent with behavioural findings of a precedence of a bias for enhanced local processing over intact global processing (Plaisted et al., 1999; Iarocci et al., 2006)). This combination of biases towards central-field locations and towards low levels of hierarchical 
processing predicts impaired response to peripherally located (lower salience) targets in conflict with centrally located (high salience) distractors, especially when such conflict occurs in low-level (high salience) properties such as orientation and colour.

Distracting stimuli can exert an unusually large effect on reaction time in people with autism (Burack, 1994), and fMRI suggests that distractor suppression in autism may evoke atypically strong activation in posterior intraparietal sulcus (Belmonte and Yurgelun-Todd, 2003b), a region also associated with suppression of visual distractors in typical individuals (Wojciulik and Kanwisher, 1999; Belmonte and Yurgelun-Todd, 2003a; Serences et al., 2005)). We applied behavioural and fMRI measures to characterise the event-related dynamics of distractor suppression in ASC, to discern whether the pattern of subclinical features in family members might extend to this non-social domain of visual perception, to ascertain whether any such behavioural distinction might be associated with differences in neurophysiology, and to determine whether the degree of correlation of functional activation amongst brain regions might differentiate family members with and without ASC. Boys with ASC, clinically unaffected brothers of people with ASC, and unrelated, typical controls performed a task demanding selective attention to colour and orientation in briefly displayed sine-wave gratings in disjoint, peripheral locations, in the presence of spatially intervening, centrally located distractors that were either congruent or incongruent to these attended stimuli (Figure 1). 


\section{Methods}

\subsection{Participants}

The study was approved by the Cambridge Local Research Ethics Committee. Informed assent was obtained from each participant, and informed consent from each participant's parent. Members of the ASC group, and the ASC sibs of members of the sib group, were identified from the Autism Research Centre volunteer pool, a database of individuals with pre-existing diagnoses of autism or Asperger syndrome made according to DSM-IV-TR (American Psychiatric Association, 2000) criteria. The volunteer pool is restricted to diagnoses made by qualified clinicians at recognised centres. Controls were recruited from local schools. General developmental delay and other neurological or neuropsychiatric conditions were excluded, as was colour-blindness. All participants had normal vision. IQ was assessed by the Wechsler Abbreviated Scale of Intelligence (WASI), except for two participants in the sib group who refused IQ testing. For members of the ASC group, the Autism Diagnostic Interview - Revised (ADI-R) (Lord et al., 1994) was conducted with one or both parents by a qualified rater (M.K.B.) ${ }^{1}$ In addition, all participants completed the adolescent version of the Autism Spectrum Quotient (AQ) (Baron-Cohen et al., 2001, 2006a), a validated screening tool (Woodbury-Smith et al., 2005) which ruled

\footnotetext{
$\overline{1}$ In order to respect the copyright on the ADI-R, the following procedure was implemented: During the interview, questions were read from one legally obtained, printed copy of the research version of the ADI-R, which was used for all administrations, and notes and codings were typed into a laptop computer. After the interview the resulting computer file, which contained only the rater's notes and codings and not the text of the ADI-R, was scored automatically by software developed by the rater. This software implements the ADI-R scoring algorithm (which is not patented) but incorporates none of the text of the ADI-R (which is copyrighted).
} 
out autism spectrum conditions in the sib group and the control group and confirmed clinical and ADI-R diagnoses in the ASC group. Although there was no formal clinical evaluation, in no case did any member of the sib group or the control group present a clinical impression of ASC.

Participants were excluded if they did not meet ADI-R diagnostic threshold or if they scored less than 32 on the AQ (in the ASC group), or if they were unable to perform the behavioural task or to tolerate the scanner environment, or if they moved excessively during the scan. One participant - the monozygotic twin of an included participant (Belmonte and Carper, 2006) - did not meet ADI-R social and communicative criteria, and one participant met ADI-R criteria by one point but did not meet the AQ criterion. One participant with ASC and two sibs were unable to perform the behavioural task. Three children with ASC, one sib, and two typical controls were unable to tolerate the scanner. Two controls moved excessively during the scan. After these exclusions, group sizes were 8 ASC, 7 sib, and 9 controls (Table 1). One sib was the brother of a member of the ASC group; the other sibs were unrelated to the ASC children enrolled in this study.

\subsection{Task}

The task required combining attention to location, colour, and orientation, and was purposefully chosen to be difficult since the interest was in maximising perceptual complexity and cognitive demand rather than performance. In order to avoid potential difficulties implementing complex verbal instructions,

verbal explanations were supplemented by visual illustrations of target and non-target stimuli along with physical rehearsals of target and non-target 
responses. Before entering the scanner, participants practised the task on a laptop computer until behavioural performance was level from one block of trials to the next, and until they felt comfortable with the task.

Each stimulus was a $3 \times 6$ array of oriented, coloured sine-wave gratings in Gabor patches, presented for 167 ms. Gratings had a spatial frequency of $4.4 /^{\circ}$, were coloured either yellow-green (hue 50 in colour space) or yelloworange (hue 95), and were oriented either $22.5^{\circ}$ or $112.5^{\circ}$ away from the horizontal ('diagonal-up' or 'diagonal-down'). Arrays were $5.5^{\circ}$ wide and $2.75^{\circ}$ tall, centred on a fixation cross $0.3^{\circ}$ in width and height. Participants were instructed to attend simultaneously to the central grating in the $3 \times 3$ segment of the array in the left hemifield and the central grating in the $3 \times 3$ segment in the right hemifield.

Imaging took place in two runs, separated by a brief rest break during which the participant remained immobile in the scanner. In the colour-orientation run, participants were instructed to press a button with the right index finger if the attended grating on the left were orange and the attended grating on the right were diagonal-up, and with the right middle finger otherwise. In the orientation-colour run, these criteria were reversed: participants pressed with the index finger if the grating on the left were diagonal-up and the grating on the right were orange, and with the middle finger otherwise. Each of the two runs comprised 166 stimulus arrays presented at $3 \mathrm{~s}$ intervals. The elements of each array were randomly chosen conjunctions of one of the two colours and one of the two orientations; these four possible combinations yielded a $25 \%$ proportion of targets. The order of the two runs was counter-balanced across participants. 
In the congruent condition, the two attended stimuli were identical to each other and to the distractors in the spatially intervening, innermost column of each hemifield, whilst the ten outer distractors remained randomly chosen. In the incongruent condition, the attended stimuli were uncorrelated with each other and with the distractors. Thus the congruent condition could be perceptually represented by collapsing colour and orientation across spatial position, whereas the incongruent condition had greater relational complexity because it required the simultaneous maintenance of these three interacting variables (colour, orientation, position). Proportions of targets and non-targets were the same in the congruent as in the incongruent condition. Participants were instructed to emphasise accuracy rather than speed, to slow themselves down so as to avoid impulsive responding, and to feel free to take as much of the $3 \mathrm{~s}$ response interval as they needed.

Autism is characterised by an abnormally strong dependence of behavioural performance on attentional and executive set: results differ markedly depending on whether instruction and expectation bias towards a strategy of locking attention onto localised features or distributing attention globally across stimuli (Plaisted et al., 1999; Iarocci et al., 2006), and trial-to-trial changes from narrow to broad attentional scope exact a heavy price in terms of behavioural performance (Mann and Walker, 2003). Because of this consideration of attentional set, our design goal was to establish a behavioural context in which the majority of stimuli demanded distribution of attention across locations and features. Each run therefore contained 34 congruent events embedded in a background of 132 incongruent events, with the constraint that no two congruent events were separated by fewer than two incongruent events. The incongruent events thus established an attention-demanding baseline response, against 
which the congruent events were negatively contrasted. Because participants had difficulty tolerating long sessions in the scanner, and because 'rest' is in any case a poorly controlled task condition when it comes to comparisons between autistic and non-autistic individuals (Kennedy et al., 2006), no rest condition was included.

\subsection{Functional Imaging}

Echo-planar images were acquired on a $3 \mathrm{~T}$ whole-body system consisting of a Bruker Medspec 30/100 spectrometer (Ettlingen, Germany) and a 910 mm bore whole-body actively shielded magnet (Oxford Magnet Technology, Oxford, UK), in 21 axial slices parallel to the AC-PC plane, with TR $1.1 \mathrm{~s}$, TE $27.5 \mathrm{~ms}$, flip angle $65.5^{\circ}$. Slices were $4 \mathrm{~mm}$ thick with a $1 \mathrm{~mm}$ gap. A 20 $\mathrm{cm}$ field of view in a 64x64 matrix yielded an in-plane resolution of $3.125 \mathrm{~mm}$. Imaging began $28.65 \mathrm{~s}$ before the onset of the first stimulus, and continued $23.35 \mathrm{~s}$ after the onset of the last stimulus.

Functional images were corrected for head motion by registering the entire image series from both runs to the last image of the first run, using Decoupled Automated Rotational and Translational motion correction (Maas et al., 1997), a method that uses a $k$-space representation of the images to separate rotational and translational ( $k$-space phase) components. fMRI time series were high-pass filtered by detrending in a moving window of 26 points (28.6 s), and temporally interpolated to correct for slice acquisition timing. 


\subsection{Statistical Analysis}

$d^{\prime}$ scores from the behavioural data were subjected to a $3 \times 2 \times 2$ (group $\times$ run $\times$ congruence) analysis of variance, with post hoc $t$ tests.

Voxel time series were correlated with an ideal hæmodynamic response to the incongruent events, computed by a balloon hæmodynamic model (Buxton et al., 1998) with the following parameters: mean venous transit time at rest $\tau_{0}=2 s$, resting oxygen extraction fraction $E_{0}=0.4$, resting blood volume $V_{0}=0.01$, maximum normalised inflow rate $f_{\max }=1.7$, outflow exponent $f_{\alpha}=0.5$, outflow linear coefficient $f_{\beta}=1.866$. Inputs to this model were set with rise time an invariant $4 \mathrm{~s}$, sustain time equal to the behavioural response latency on each trial, and instantaneous fall time. These input parameters to the balloon model yielded a smooth output peaking near 5.5s and decaying near zero within $9 \mathrm{~s}$ (a curve similar to a standard $\gamma$ variate, but based on an explicit physical model and accounting for trial-to-trial variation in response latency). Overlapping responses were summed. Only the events associated with correct responses were included in this analysis; intervals containing hæmodynamic responses associated with incorrect behavioural responses were specifically excluded from the correlation computation. ${ }^{2}$

The resulting correlation images were transformed to Talairach space (Talairach and Tournoux, 1988) with interpolation to $1 \mathrm{~mm}^{2}$ voxels, and convolved with a $6.25 \mathrm{~mm}$ FWHM Gaussian kernel. To identify structures activated within each of the three participant groups considered separately, a voxelwise one-sample $t$ test was thresholded at a two-tailed probability of 0.01 and a cluster size of 470

$\overline{2}$ In every participant sufficient trials for averaging remained after exclusion of incorrect responses - see the behavioural accuracy figures. 
$\mu l .{ }^{3}$ Activated structures were labelled by hand with reference to automated labellings generated by the Talairach Dæmon (Lancaster et al., 2000). To identify structures differentially activated in the three groups, between-groups $t$ tests were applied only to those voxels significantly activated or deactivated in any of the three within-group tests. These $t$ images were thresholded at a two-tailed probability of 0.05 .

To explore activations across time as well as across space, average fMRI responses were computed for each participant. At each voxel in the Talairachtransformed image, and for incongruent and congruent events separately, the 17 scans (18.7 s) following an event were averaged temporally across events and spatially over a radius of $3.125 \mathrm{~mm}$, and normalised as percent signal change from time zero. ${ }^{4}$ The congruent average was subtracted from the incongruent average to form a difference average consisting only of the BOLD effect associated with decreased perceptual congruence. (This subtraction method is analogous to the computation of a 'difference wave' in the context of eventrelated potentials.) To reduce noise, each of these single-participant averages underwent two iterations of three-point smoothing. Pointwise $t$ tests compared these difference averages across groups, and grand averages were computed for each of the three participant groups.

In any clinical study, confounds of diagnostic group with behavioural performance can be difficult to avoid, and the directionality of the relationship between

$\overline{3}$ According to an empirical null distribution of maximum cluster sizes computed by $t$ test over 7 independent null data sets with the identical $6.25 \mathrm{~mm}$ FWHM blurring, these voxel-probability and cluster-size constraints implemented a cluster-level tail probability of 0.05 .

4 Though $18.7 \mathrm{~s}$ is a much longer averaging epoch than is typical in event-related fMRI studies, this interval was necessary in order to capture the longer-lasting, secondary modulations induced in the two experimental groups. 
behaviour and physiology can be unclear. Even our exclusion of trials with incorrect behavioural responses still allows some possibility that observed group differences in physiology might have been induced by differences in behavioural performance. To evaluate this possibility, an index of the 'abnormality' of BOLD response was computed, and regressed against $d^{\prime}$ score: First, individual fMRI time series within each region of interest were correlated against the group grand average within that region, for each individual in each of the ASC and sib groups. That is to say, each ASC time series was correlated against the ASC grand average, and each sib was correlated against the sib grand average. In addition, these same individual averages in the ASC and sib groups were correlated against the grand average from the normal group. After Fisher's z transformation, the individual's correlation against the normal time series was subtracted from the individual's correlation against their own group's time series, yielding a normally distributed measure of where each individual lay on a continuum between identity with the normal time series and identity with their own group's abnormal (ASC or sib) time series. These abnormality indices were then regressed against $d^{\prime}$ scores from the incongruent condition, using either linear or quadratic regression, whichever made the best fit in each case. Within the sib and ASC groups, a positive effect of $d^{\prime}$ on the abnormality index would indicate that those individuals who were most driving the observed abnormal group time series were those with the most normal behaviour, whereas a negative effect would indicate that the abnormal physiological effect were associated with abnormal behaviour, and the absence of any measurable effect would suggest that degrees of physiological and behavioural abnormality were not strongly associated.

For an exploratory, hypothesis-generating evaluation of functional correlation 
between brain regions, a set of 38 bilateral (76 total) regions of interest (Table 2) was identified by selecting all regions activated in any of the group $t$ images, as well as several regions activated just below the cluster-size threshold. Correlation matrices for averaged time series in all these regions were computed within each participant separately, and converted to $z^{\prime}$ scores by Fisher's transformation. $z^{\prime}$ scores were compared using $t$ tests within each region pair in the correlation matrix, both within and between groups. In addition, $z^{\prime}$ scores pooled across region pairs were compared between groups using an analysis of variance and post hoc $t$ tests, with degrees of freedom adjusted to reflect the number of regions (that is, the number of independent observations) rather than the number of region pairs.

To relate levels of autistic traits to levels of functional activation within brain regions, the normality index ( $z$-transformed correlation with the normal group average fMRI time series, as above) was modelled as a function of AQ score in each of four bilateral, distinct regions of interest (see Table 2) along the length of each hemisphere's middle frontal gyrus, from Brodmann area 10 near area 46, to area 9 near area 8 (SAS PROC MIXED with fixed effects of AQ, group, hemisphere and region, and random effect of participant). Within the ASC group, ADI-R social, communicative and repetitive behaviours subscores each were similarly modelled (SAS PROC MIXED with fixed effects of ADI-R, hemisphere and region, and random effect of participant).

To relate levels of autistic traits to levels of functional correlation between brain regions, $z^{\prime}$ for functional correlations (computed as above), averaged within each participant across all pairs of regions, was modelled as a function of $d^{\prime}$ from the behavioural task and as a function of AQ score (SAS PROC MIXED with fixed effects of $d^{\prime}$ or AQ and group, and random effect of participant). 
In the ASC group, $z^{\prime}$ was also modelled as a function of ADI-R social, communicative, and repetitive behaviour subscores (SAS PROC MIXED with fixed effects of ADI-R subscore and random effect of participant).

\section{$3 \quad$ Results}

\subsection{Behaviour}

Despite an instruction to focus on accuracy rather than speed, mean response time for the ASC group was slightly but significantly shorter than those for the typical and sib groups: $753 \pm 34 \mathrm{~ms}$ versus $889 \pm 35 \mathrm{~ms}$ and $918 \pm 52 \mathrm{~ms}$ (mean \pm S.E.M.), respectively $(\mathrm{F}(2,84)=4.40, \mathrm{p}=0.0152)$. Accuracy, the behavioural variable of interest, showed a pattern of differences distinct from that of the reaction times (Figure 2): $d^{\prime}$ scores differed significantly by diagnosis $(\mathrm{F}(2,84)$ $=5.99, \mathrm{p}=0.0037)$, being lower than normal in the sib group $(\mathrm{t}(62)=2.36, \mathrm{p}$ $=0.0217)$, and lowest in the ASC group $(\mathrm{t}(66)=3.42, \mathrm{p}=0.0011)$. (In terms

of the proportion of correctly classified stimuli, accuracy for incongruent and congruent conditions, respectively, was $80 \%$ and $84 \%$ in the normal group, $71 \%$ and $76 \%$ in the sib group, and $68 \%$ and $74 \%$ in the ASC group.) Thus, in terms of this behavioural measure, the sibs' response partially mimicked that of ASC.

In order to ensure that these effects were not being driven by possible outliers, the analyses were re-run excluding the two participants in the sib group with the largest VIQ-PIQ discrepancies and the participant in the ASC group with the lowest PIQ and the lowest $d^{\prime}$. Response time differences were again significant but slight $(799 \pm 29 \mathrm{~ms}$ versus $889 \pm 35 \mathrm{~ms}$ and $1021 \pm 50 \mathrm{~ms}, \mathrm{~F}(2,72)$ 
$=6.76, \mathrm{p}=0.002) . d^{\prime}$ again differed significantly by diagnosis $(\mathrm{F}(2,72)=3.86$, 0.0255), but with this exclusion of outliers from the sib group the $d^{\prime}$ difference was driven only by the ASC group $(\mathrm{t}(62)=2.65, \mathrm{p}=0.0102)$. In addition, with this exclusion of outliers the expected main effect of congruence appeared, $\mathrm{F}(1,72)=4.53, \mathrm{p}=0.0367$, with $d^{\prime}$ greater in the congruent condition than in the incongruent condition.

\subsection{Functional Mapping: Typical Controls}

Details of event-related activation foci within each group and in contrasts between groups are presented in Table 3, and representative group images in Figure 3. In controls, low congruence activated a distributed network of regions including an attention-related region in left anterior lateral cerebellum (Allen et al., 1997) ${ }^{5}$, bilateral middle frontal gyri, dorsal anterior cingulum, right anterior intraparietal sulcus, right supramarginal gyrus, and left superior parietal lobule. Also activated were left mediodorsal thalamus, left parahippocampal gyrus, right lateral cuneus, rostral anterior cingulum, left anterior insula, and a location in left medial fusiform gyrus consistent with the location of the V8 colour region (Hadjikhani et al., 1998).

\subsection{Functional Mapping: $A S C$}

Comparing regions of statistically significant activation (see Table 3 for $t$ values and cluster extents), the ASC group was notable for atypical frontal and

$\overline{5}$ In 2 controls, 3 particpants with ASC, and 3 sibs the cerebellar attention area lay just beyond the imaged volume and could not be evaluated. The result remains statistically significant, though, even given this reduced number of observations. 
superior parietal activations including a lack of activation in dorsal anterior cingulum and lack of significant activation in left anterior insula, left middle frontal gyrus, and superior parietal lobe. In addition, activations arising within the autism group but not significant in the between-groups comparison included extensive cerebellar activation centred in the left pyramis, activation of orbitofrontal cortex, right posterior superior temporal gyrus, and (as predicted) the posterior extent of right intraparietal sulcus near its junction with the transverse occipital sulcus, a localisation consistent with the representation of parafoveal space along the medial wall of the intraparietal sulcus (between foveal space at the fundus and far peripheral space on the adjacent gyrus) within area V7 (Swisher et al., 2007).

\subsection{Functional Mapping: Sibs}

The sib group was remarkable for what seemed an overall lack of significant activations - at least in correlation with our activation model validated in a normal pilot group. The only regions significantly differentially active in sibs in this contrast between high and low congruence were rostral anterior cingulum and also the retrosplenial portion of posterior cingulum, a region associated with establishing spatial expectancy (Small et al., 2003).

\subsection{Time Courses: Primary Phase}

Examination of time courses for all regions activated in any of the groups revealed several frontal, cerebellar, and occipitoparietal regions (Figure 5) where congruence in the current event affected BOLD responses during the 
events immediately subsequent, in the ASC and sib groups. The resulting abnormally prolonged BOLD responses therefore were not detected by our model based on normal pilot data. In general, these responses occurred in two phases, a primary phase from 0 to $7 \mathrm{~s}$ and a secondary and overlapping phase from $7 \mathrm{~s}$ to about $18 \mathrm{~s}$.

In the primary phase, the typical group tended to activate dorsal anterior cingulum more than the ASC group $(t(15)=2.04, p=0.059)$, and amongst the sibs there was considerable variation with no clear pattern (no significant contrasts between sibs and ASC nor between sibs and normal). In contrast, all three groups activated bilateral middle frontal gyri. Interestingly, in the cerebellar attention area sibs activated more strongly $(t(9)=2.50, p=0.034)$ whilst ASC did not differ significantly from controls. Although these cingulate and cerebellar activatons distinguished the ASC and sib groups from controls, occipitoparietal regions were more variable across participants and no significant group differences were found.

After exclusion of the aforementioned two sib and one ASC behavioural outliers, re-running this analysis maintained or augmented all the results: for dorsal anterior cingulum, controls $>$ ASC, $t(14)=2.65, p=0.019$ and also sibs $>$ controls, $t(12)=2.26, p=0.044$ and sibs $>$ ASC, $t(10)=4.10, p=0.0022$; for the cerebellar attention area, sibs $>$ controls, $t(8)=3.16, p=0.0133$ and also controls $>$ ASC, $t(9)=2.31, p=0.047$. 


\subsection{Time Courses: Secondary Phase}

The secondary phase extended well past the usual decay period of hæmodynamic response to a single trial - that is, this phase consisted of altered responses not to the event of interest, but rather to events immediately succeeding the event of interest: the manipulation of congruence within the initial event induced a longer-lasting change in the brain response to subsequent events. This unusual response arose in $\mathrm{ASC}$ in the cerebellar attention area $(t(10)=3.98, p=$ 0.0026). The sibs manifested a similar pattern of prolonged activations, with a stronger effect that was significant in a larger set of regions. Sibs activated more strongly than controls in the cerebellar attention area $(t(9)=6.25, p=$ $0.00015)$, left $(t(14)=3.18, p=0.0067)$ and right $(t(14)=2.25, p=0.041)$ middle frontal gyri, and right posterior intraparietal sulcus $(t(14)=3.75, p=$ 0.0022). In all these regions, the response in sibs was even greater than that in ASC.

Again after exclusion of the behvioural outliers, these results were maintained. The ASC group again activated the cerebellar attention area more than normal, $t(9)=3.13, p=0.012$. In the sibs, contrasts were significant for greater activations in the cerebellar attention area $(t(8)=7.51, p=0.000069)$, left middle frontal gyrus $(t(12)=3.73, p=0.0029)$ with a like trend in right middle frontal gyrus $(t(12)=1.99, p=0.070)$, and right posterior intraparietal sulcus $(t(12)=3.39, p=0.0054)$. 


\subsection{Relation of Behavioural and Physiological Differences}

Sibs showed a strong quadratic relationship between $d^{\prime}$ and atypicality of the fMRI time series in bilteral middle frontal gyri $(t(4)=+3.43$ and $p=$ 0.0264, same values in both gyri separately), as well as a trend towards a similar quadratic relationship in right posterior intraparietal sulcus $(t(4)=$ $+2.41, p=0.0733)$. The ASC group showed a quadratic relationship in dorsal anterior cingulum $(t(5)=+3.95, p=0.0108)$ and a borderline negative linear relationship in right posterior intraparietal sulcus $(t(6)=-2.42, p=0.0515)$ (Figure 4).

\subsection{Functional Correlations between Regions}

Though no between-groups comparisons of functional correlations within individual region pairs survived Bonferroni correction for multiple comparisons, functional correlation pooled across all possible pairs of regions of interest was significantly lower in ASC than in the other two groups (omnibus $F(2,1821)=266.89$, post $\operatorname{hoc} t(1291)=20.71$ between ASC and controls, $t(1139)=19.84$ between ASC and sibs, but $t(1215)=-0.72$ between sibs and controls).

Exclusion of the behavioural outliers strengthened the large difference between ASC and the other two groups (omnibus $F(2,1593)=308.19$, post hoc $t(1215)=$ 21.04 between ASC and controls, $t(913)=22.55$ between ASC and sibs), and also revealed a comparatively slight yet statistically significant difference between sibs and controls $(t(1065)=-4.53)$, in a direction opposite to that of the contrast between ASC and controls: that is, in this omnibus test in which co-activation data were collapsed across all possible pairings of brain 
regions, the five sibs remaining after exclusion of two behavioural outlier sibs manifested functional co-activation slightly greater than controls', unlike the ASC probands in whom functional co-activation was markedly less than controls'. (Despite this increase in overall co-activation across all pairs, the exclusion of outliers actually produced fewer individual pairs of regions that reached the uncorrected significance threshold $\alpha=0.05$, owing to the decrease in the number of observations in the sib sample.)

Figure 6 presents graphically the results of comparisons within region pairs, uncorrected for multiple comparisons and (for conservatism) without exclusion of outliers. Most evident in the control group was a strong coupling between dorsal anterior cingulum, cerebellum, superior parietal lobule, and lingual gyrus and other occipital regions. This group also manifested strong correlations within a visual processing network comprising fusiform gyrus, cuneus, precuneus, and posterior superior temporal gyrus, a stimulus evaluation network comprising superior temporal gyrus, insula, and inferior frontal gyrus, and a response network comprising middle frontal gyrus, dorsal anterior cingulum, and left medial and precentral gyri. The ASC group, in contrast, was typified by correlations between cuneus, precuneus, and middle frontal gyrus, and quiescence of dorsal anterior cingulum. Significant correlations in ASC also existed between middle frontal gyrus and rostral anterior cingulum and orbitofrontal cortex, regions involved in affective control. The complete sib group activated a dorsal anterior cingulum network similar to that of the control group, albeit one with lesser correlation with middle frontal gyrus and superior parietal lobule. 


\subsection{Relation to Psychometric Measures of Autistic Traits}

In the model of normality of brain activation as a function of AQ score, $t$ scores for all middle frontal gyrus (MFG) regions in all participant groups including the control group - were negative. This relationship was significant in posterior MFG in ASC (left MFG region \#4 Kenward-Roger $t(45.7)=$ $-2.09, p=0.0421$; right $\mathrm{MFG}$ region $\# 3 t(45.7)=-2.22, p=0.0315)$, and in anterior and posterior MFG in sibs (region \#1 left and right $t(56.6)=$ $-3.56, p=0.0008$ and $t(56.6)=-2.00, p=0.0502$, respectively; region \#3 left and right $t(56.6)=-3.21, p=0.0022$ and $t(56.6)=-2.11, p=0.0392$; region \#4 left and right $t(56.6)=-2.48, p=0.0160$ and $t(56.6)=-4.29, p<$ 0.0001). Similarly, in the ASC group $t$ scores for all three ADI-R subscores in all MFG regions were negative; ADI-R social and communicative subscores were strongly negatively associated with the normality index in most regions of posterior MFG (left MFG region \#3 social $t(55)=-2.12, p=0.0388$, communicative $t(55)=-2.07, p=0.0432$; right MFG region \#3 social and communicative both $t(55)=-2.93, p=0.0049$; left MFG region \#4 social $t(55)=-2.73, p=0.0085$, communicative $t(55)=-2.92, p=0.0051)$, and trended more weakly in anterior MFG (left MFG region \#1 social $t(55)=$ $-2.18, p=0.0335$, communicative $t(55)=-2.10, p=0.0404 ;$ right MFG region $\# 1$ social $t(55)=-1.99, p=0.0511$, communicative $t(55)=-1.89, p=$ 0.0636). (See Table 2 for Talairach coordinates.) There were no significant associations with the ADI-R repetitive behaviours subscore, although in this case again all eight $t$ scores (independent tests in four bilateral, distinct regions) were negative.

In the models of within-participant mean functional correlation as a function 
of behavioural $d^{\prime}$, AQ score and ADI-R subscores, the only significant effect and only marginally so - was that of AQ in the ASC group (Kenward-Roger $t(15.5)=-2.06, p=0.0567)$. Functional correlation was unrelated to any of these variables in the control or sib groups, and unrelated to $d^{\prime}$ or ADI-R in the ASC group.

\section{Discussion}

Behaviourally, although the ASC group responded more quickly than either the controls or the sibs, the ASC group was less accurate than the sibs, who in turn were less accurate than the controls. This finding of autistic impairment in a spatial divided-attention task echoes psychophysical and electrophysiological

observations of an atypical spatial distribution of visual attention in autism (Townsend et al., 1994).

Physiologically, incongruence-related activations in the controls occupied a widespread network of frontal, cerebellar, and parietal attention regions, whereas the ASC group activated a cerebellar region outside the attention area, did not phasically activate frontal and parietal attention regions, but did activate posterior visual regions and also orbitofrontal cortex. These findings appear to confirm a large number of previous results from various cognitive tasks, suggesting hypoactivation of frontal cortices in autism and hyperactivation of posterior cortices subserving lower levels of processing (Haist et al., 2005; Silk et al., 2006; Belmonte et al., 2004b), as well as results on orbitofrontal activation possibly related to arousal (Belmonte and Yurgelun-Todd, 2003b), anterior cingulate hypoactivation (Mundy et al., 2003; Gomot et al., 2006), and hypoactivation in the cerebellar attention region with hyperactivation of 
other cerebellar regions (Allen and Courchesne, 2003; Allen et al., 2004). In the sibs, in contrast, differential activations between the congruence conditions were limited to only two regions: rostral anterior cingulum and retrosplenial cortex.

These modelled activations, however, do not tell the whole story. Atypical activation maps may arise because the activation really isn't there, or because the activation is atypically timed and fails to onset and/or to resolve within the modelled time interval. Examination of time courses revealed ASC and sib activations whose atypically delayed and prolonged timing had prevented detection in the whole-brain analysis. ${ }^{6}$ That is, the ASC and sib groups did activate fronto-cerebellar attention systems, but these activations arose too late to be useful during behavioural response to the trial of interest, instead manifesting during the trials immediately subsequent. ${ }^{7}$

It is notable that sibs do not attain normative levels of performance: the difference in BOLD response in a direction aligned with that of behavioural performance between the sib and ASC groups but still opposite that of behavioural performance between the sib and control groups is what suggests the operation of a compensatory process. Also of interest is that these delayed activations are even greater in magnitude in sibs than in ASC, suggesting that the sibs may more efficiently or completely implement this putative compensatory process. This difference may reflect either the absence in the sibs of some of the genetic

$\overline{6}$ Our chosen contrast cannot, of course, distinguish between hyperactivation following incongruent trials and hypoactivation following congruent trials. In either case, though, the resemblance between ASC and sibs is of interest.

7 Note that our constraint on the separation of congruent and incongruent events allows us to meaningfully subtract responses up to two trials out. The resulting 9 s interval (current trial plus two subsequent trials) includes the onset of the second phase of the response. 
or environmental factors that confer liability to autism, or the presence of genetic or environmental factors that protect against autism.

These findings of differential timing form an important counterpoint to the oft-repeated contention that people with autism simply do not activate many of the same brain regions activated in controls. This delayed response is consistent with delayed activation in an evoked-potentials study of response to peripheral visual stimuli (Townsend et al., 2001), and with studies of attention shifting showing that impaired performance normalises when two to three seconds are allowed in which to accomplish the shift (Townsend et al., 1996). It is also consistent with many clinical behavioural observations and self-reports highlighting the importance of having explicit instruction and time to prepare responses to unpredictable stimuli.

It is important to note that the ASC group's pattern of prolonged response could not have been directly associated with motor activation, since the ASC group's motor responses were faster than normal. Group differences in accuracy also are unlikely to be a causal factor in these physiological differences, since only the trials associated with correct behavioural responses were included in the averages. It is of course possible that group differences in impulse control, distractibility and concentration may be seen as a confound in the current results, if such differences are not viewed as part of the syndrome of ASC. In any case, although the group differences in brain response that we have presented might permit stronger inferences in the context of equal behavioural responses between groups, such behavioural equality is often not the case in studies of autism and other clinical populations, and brain differences may cause behavioural differences just as well as the converse. 
The quadratic effect of $d^{\prime}$ on the abnormality of fMRI time series in key prefrontal and posterior brain regions suggests that at least in the sibs, the group's atypical physiology was not driven solely by abnormally low behavioural performers - indeed, Figure 4 illustrates that the participants with the greatest $d^{\prime}$ scores, those that fell within the normal range (see Table 1), were amongst those with the most delayed and prolonged physiological responses - a relationship verified by inspection of each individual participant's time series in comparison to the group and normal control averages. The quadratic nature of this relationship further suggests the intriguing possibility of a heterogeneous effect of atypical physiology on behaviour, one in which heightened prefrontal activation can either succeed or fail in compensating for atypical neural and cognitive processes. With regard to right intraparietal sulcus in particular, it is interesting to speculate that the negative linear effect in ASC might be akin to the left side of the sibs' quadratic effect.

The negative $t$ scores in all four bilateral (eight total), distinct middle frontal gyrus (MFG) regions in all three groups suggest a general relationship between $\mathrm{AQ}$ and frontal activation: the greater the AQ, the more delayed and prolonged the fMRI time course. This relationship manifested in posterior MFG (Brodmann area 9) for the ASC and sib groups, and also in anterior MFG for the sib group. Within the ASC group, ADI-R subscores for social and communicative but not repetitive behaviours were similarly related to MFG atypicality. These relationships to social and communicative impairments arise in the context of a non-social task of visual attention, suggesting a common substrate for social and non-social applications of cognitive control. Such a commonality between social and non-social domains makes sense in the context of Minshew et al.'s notion of a domain-general impairment in complex processing, and in the 
context of abnormal neural connectivity which has been suggested as a basis for that impairment. It also fits with Dawson et al.'s notion of perceptual abnormality as a contributing cause of autistic social deficits, and with the possibility of remediating those deficits by slowing the physical presentation of social stimuli (Tardif et al., 2007) to match the slowed pace of frontal activation.

A limit to this study is the lack of gaze tracking during performance of the task. Gaze monitoring within the scanner environment was a difficult technical obstacle at the time these data were collected, and without gaze data one cannot absolutely rule out the possibility that group behavioural and neurophysiological differences may have been driven by group differences in eye direction. However, a combination of features of the experimental paradigm, the analytical procedure, and the brain activation data seem to render this possibility unlikely: since stimuli were presented only for $167 \mathrm{~ms}$, participants would not have had time to implement saccades away from the stimulus location; furthermore, since the analysis included only the trials associated with correct responses, during these analysed trials participants were likely to have been looking at the stimulus location when the stimulus was presented; and in addition, the ASC group's strong activations in the visual regions left fusiform gyrus and right posterior intraparietal sulcus (V7) suggest that visual stimuli were indeed being processed during these trials.

The qualitative resemblances between ASC and sibs that we have presented, though interesting, raise a troubling question: if clinically unaffected sibs of people with ASC are 'slightly autistic' on these behavioural and physiological measures of visual attention (as has already been shown in the case of higherlevel social cognitive measures (Constantino et al., 2006)), then what is it 
that makes some people in these families autistic and some not? As noted above, we and many others have suggested that functional connectivity may be an important metric in autism. We found anatomically distributed patterns of functional correlation in the control and sib groups, including a visual attention network comprising many frontal, cerebellar, parietal, and occipital regions. The ASC group, in contrast, manifested overall much lower functional correlation (in an analysis pooled across all region pairs), and what correlation did exist seemed concentrated (in an exploratory analysis uncorrected for multiple comparisons) between middle frontal gyrus, cuneus and precuneus - a pattern that seemingly bypasses most of the frontal processing resources brought to bear in the control and sib groups, and is consistent with a recent result on fronto-posterior connectivity in ASC during a task of facial emotion recognition (Wicker et al., 2008).

This result of reduced inter-regional correlation is consistent with a previous finding of high variability and low correlation of resting-state metabolism between brain regions (Horwitz et al., 1988), and with recent results of decreased functional connectivity in autism during a variety of cognitive tasks (Just et al., 2004; Koshino et al., 2005; Kana et al., 2006; Just et al., 2007). Importantly, although the sibs did resemble the ASC group in terms of activations, they did not measurably resemble the ASC group in terms of overall inter-correlation. If there is any bifurcation in the developmental landscape, beyond which one path converges to autism and another escapes it, the current study suggests that this distinction may be most expressed in terms of long-range functional correlations, reflecting co-activation between brain regions. Activity within regions, on the other hand, may form more of a continuum in keeping with the behavioural measures of this and other studies, and may be permissive of 
ASC but not determinative. As attention is known to act at least in part by modulating functional connectivity between brain regions (Friston \& Büchel, 2000; Wang et al., in press), perhaps the particularly strong (albeit abnormally timed) activations in non-ASC sibs in brain regions associated with attentional/executive functions may be what preserves functional connectivity in these sibs, rescuing them from the ASC phenotype.

A perennial question in fMRI studies of ASC is the extent to which the highfunctioning subpopulation that is capable of tolerating the scanner environment may generalise to the wider ASC population. This is an especial concern in the context of the current study, where six of the eight participants with ASC had VIQ > PIQ, with two in the very superior range of VIQ. Overall, IQ measures in the ASC group had four times the variance of those in the other two groups, and even by this larger variance measure mean VIQ and PIQ in the ASC group were half a standard deviation lower than in the other two groups. Despite this peculiarity of our sample, the results seem consistent with previous findings of altered attention and altered patterns of brain activation and brain connectivity in autism, and are at least useful in establishing a hypothesis of familial patterns of atypical fronto-cerebellar activation and more ASC-specific patterns of decreased functional correlation.

It is also important to note that the alterations in functional co-activation that we have desribed do not necessarily reflect alterations in functional connectivity. Whereas functional connectivity as measured by fMRI was originally described as low-frequency signal correlation during rest, our measure of functional

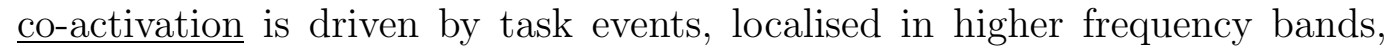
and dependent on the identification of activated brain regions. Our result of decreased co-activation in the ASC group therefore may reflect a significantly 
lower number of activated regions, a significantly greater variability in the anatomical loci of activation across individuals, or atypically low functional connectivity - all of which findings have been reported in autism (Müller, 2007). Although sorting out these potential mechanisms of apparently decreased co-activation remains a topic for further study, the specificity of this effect to $\mathrm{ASC}$ and its absence in the sib group may prove to be a significant clue as to the process of autistic brain development.

The current study establishes several imperatives for future neurophysiological investigations of autism. First, familial endophenotypes are significant and span task domains and levels of analysis. Much can be gained from combining behavioural and physiological methods, correlating across social and non-social task domains, and comparing ASC and non-ASC family members. At the time of this writing, only two other functional imaging studies have examined clinically unaffected family members: Baron-Cohen et al. (2006b) studied parents of children with ASC and reported preliminary findings of hypoactivation of extrastriate cortex during the Embedded Figures Test and hyperactivation of left inferior frontal gyrus during the Reading the Mind in the Eyes Test; however, as this study included neither an ASC comparison group nor an age-matched control group, it is difficult to make inferences regarding group similarities. Dalton et al. (2007) studied sibs in an age range slightly broader than this study's, and found decreased amygdala volume, along with decreased gaze fixation on eyes and decreased activation of right fusiform gyrus during a face recognition task. Future studies of brain activation and functional connectivity might very usefully address the possible relationships between such social perceptual characteristics and non-social traits, in family members with and without ASC. Second, in studies of autistic functional anatomy - 
as in studies of developmental disorders in general (Johnson et al., 2002)differences in the timing of activations may be even more important than differences in their anatomical loci, and models based on normal time courses may miss out the most interesting results. In future, model-free analyses using multivariate methods such as partial least squares or independent components analysis will be crucial to such investigations. Third, both abnormalities in regional time courses and abnormalities in inter-regional functional connectivity may be more precisely characterised by complementing fMRI with more temporally specific methods such as quantitative EEG (Brock et al., 2002) and MEG. These techniques benefit from recent advances in high-density recording hardware and multivariate analysis. We hope that autism research as a field will take up these imperatives, as we intend to do.

\section{Acknowledgements}

M.K.B. was funded by a Young Investigator Award from Cure Autism Now, and M.G. by grants from the Fondation pour la Recherche Médicale, the Région Centre, and the Fondation d'Entreprise France Telecom. MRI scanning took place at the Wolfson Brain Imaging Centre, Cambridge, and was funded by an MRC grant awarded to Simon Baron-Cohen. We thank the MIT Student Information Processing Board for providing computing facilities, and in particular Alex Rolfe for helping with disaster recovery. Most of all, thanks are due to our participants and their families for their time and enthusiasm. 


\section{References}

Allen, G., Buxton, R.B., Wong, E.C., \& Courchesne, E. (1997). Attentional activation of the cerebellum independent of motor involvement. Science, 275, 1940-1943.

Allen, G., \& Courchesne, E. (2003). Differential effects of developmental cerebellar abnormality on cognitive and motor functions in the cerebellum: an fMRI study of autism. American Journal of Psychiatry, 160, 262-273.

Allen, G., Müller, R.-A., \& Courchesne, E. (2004). Cerebellar function in autism: functional magnetic resonance image activation during a simple motor task. Biological Psychiatry, 56, 269-278.

American Psychiatric Association (2000). Diagnostic and Statistical Manual of Mental Disorders 4/e (Text Revision). Washington: American Psychiatric Association.

Andrews, G., Halford, G.S., Bunch, K.M., Bowden, D., \& Jones, T. (2003). Theory of mind and relational complexity. Child Development, 74, 14761499.

Baron-Cohen, S., \& Hammer, J. (1997). Parents of children with Asperger syndrome: What is the cognitive phenotype? Journal of Cognitive Neuroscience, $\mathbf{9}, 548-554$.

Baron-Cohen, S., Hoekstra, R.A., Knickmeyer, R.C., \& Wheelwright, S. (2006a). The Autism-Spectrum Quotient (AQ) - adolescent version. Journal of Autism and Developmental Disorders, 36, 343-350.

Baron-Cohen, S., Ring, H.A., Chitnis, X., Wheelwright, S., Gregory, L.J., Williams, S.C.R., Brammer, M.J., \& Bullmore, E.T. (2006b). fMRI of parents of children with Asperger syndrome: a pilot study. Brain and Cognition, 61, 122-130. 
Baron-Cohen, S., Wheelwright, S., Skinner, R., Martin, J., \& Clubley, E. (2001). The Autism-Spectrum Quotient (AQ): evidence from Asperger syndrome / high-functioning autism, males and females, scientists and mathematicians. Journal of Autism and Developmental Disorders, 31, 517.

Belmonte, M.K. (2000). Abnormal attention in autism shown by steady-state visual evoked potentials. Autism, 4, 269-285.

Belmonte, M.K., Allen, G., Beckel-Mitchener, A., Boulanger, L.M., Carper, R.A., \& Webb, S.J. (2004a). Autism and abnormal development of brain connectivity. Journal of Neuroscience, 24, 9228-9231.

Belmonte, M.K., \& Carper, R.A. (2006). Monozygotic twins with Asperger syndrome: differences in behaviour reflect variations in brain structure and function. Brain and Cognition, 61, 110-121.

Belmonte, M.K., Cook, E.H. Jr., Anderson, G.M., Rubenstein, J.L.R., Greenough, W.T., Beckel-Mitchener, A., Courchesne, E., Boulanger, L.M., Powell, S.B., Levitt, P.R., Perry, E.K., Jiang, Y., DeLorey, T.M., \& Tierney, E. (2004b). Autism as a disorder of neural information processing: directions for research and targets for therapy. Molecular Psychiatry, 9, 646-663. Unabridged edition at http://www . cureautismnow .org/conferences/summitmeetings/

Belmonte, M.K., \& Yurgelun-Todd, D.A. (2003a). Anatomic dissociation of selective and suppressive processes in visual attention. NeuroImage, 19, 180189.

Belmonte, M.K., \& Yurgelun-Todd, D.A., 2003b. Functional anatomy of impaired selective attention and compensatory processing in autism. Cognitive Brain Research, 17, 651-664.

Bertone, A., Mottron, L., Jelenic, P., \& Faubert, J. (2003). Motion perception 
in autism: a "complex" issue. Journal of Cognitive Neuroscience, 15, 218225.

Bertone, A., Mottron, L., Jelenic, P., \& Faubert, J. (2005). Enhanced and diminished visuo-spatial information processing in autism depends on stimulus complexity. Brain, 128, 2430-2441.

Brock, J., Brown, C.C., Boucher, J., \& Rippon, G. (2002). The temporal binding deficit hypothesis of autism. Development and Psychopathology, 14, 209-224.

Burack, J.A. (1994). Selective attention deficits in persons with autism: preliminary evidence of an inefficient attentional lens. Journal of Abnormal Psychology, 103, 535-543.

Buxton, R.B., Wong, E.C., \& Frank, L.R. (1998). Dynamics of blood flow and oxygenation changes during brain activation: the balloon model. Magnetic Resonance in Medicine, 39, 855-864.

Constantino, J.N., Lajonchere, C., Lutz, M., Gray, T., Abbacchi, A., McKenna, K., Singh, D., \& Todd, R.D. (2006). Autistic social impairment in the siblings of children with pervasive developmental disorders. American Journal of Psychiatry, 163, 294-296.

Constantino, J.N., \& Todd, R.D. (2003). Autistic traits in the general population: a twin study. Archives of General Psychiatry, 60, 524-530.

Courchesne, E., \& Pierce, K. (2005). Why the frontal cortex in autism might be talking only to itself: local over-connectivity but long-distance disconnection. Current Opinion in Neurobiology, 15, 225-230.

Dalton, K.M., Nacewicz, B.M., Alexander, A.L., \& Davidson, R.J. (2007). Gaze-fixation, brain activation, and amygdala volume in unaffected siblings of individuals with autism. Biological Psychiatry, 61, 512-520.

Dawson, G., Webb, S.J., Wijsman, E., Schellenberg, G., Estes, A., Munson, 
J., \& Faja, S. (2005). Neurocognitive and electrophysiological evidence of altered face processing in parents of children with autism: implications for a model of abnormal development of social brain circuitry in autism. Development and Psychopathology, 17, 679-697.

Friston, K.J., \& Büchel, C. (2000). Attentional modulation of effective connectivity from V2 to V5/MT in humans. Proceedings of the National Academy of Sciences of the United States of America, 97, 7591-7596.

Gomot, M., Bernard, F.A., Davis, M.H., Belmonte, M.K., Ashwin, C., Bullmore, E.T., \& Baron-Cohen, S. (2006). Change detection in children with autism: an auditory event-related fMRI study. NeuroImage, 29, 475484.

Hadjikhani, N., Liu, A.K., Dale, A.M., Cavanagh, P., \& Tootell, R.B.H. (1998). Retinotopy and color sensitivity in human visual cortical area V8. Nature Neuroscience, 1, 235-241.

Haist, F., Adamo, M., Westerfield, M., Courchesne, E., \& Townsend, J. (2005). The functional neuroanatomy of spatial attention in autism spectrum disorder. Developmental Neuropsychology, 27, 425-458.

Halford, G., Wilson, W.H., \& Phillips, S. (1998). Processing capacity defined by relational complexity: implications for comparative, developmental, and cognitive psychology. Behavioral and Brain Sciences, 21, 803-831.

Happé, F., Briskman, J., \& Frith, U. (2001). Exploring the cognitive phenotype of autism: weak "central coherence" in parents and siblings of children with autism: I. Experimental tests. Journal of Child Psychology and Psychiatry, 42, 299-307.

Horwitz, B., Rumsey, J.M., Grady, C.L., \& Rapoport, S.I. (1988). The cerebral metabolic landscape in autism. Intercorrelations of regional glucose utilization. Archives of Neurology, 45, 749-755. 
Iarocci, G., Burack, J.A., Shore, D.I., Mottron, L., \& Enns, J.T. (2006). Global-local visual processing in high functioning children with autism: structural vs. implicit task biases. Journal of Autism and Developmental Disorders, 36, 117-129.

Johnson, M.H., Halit, H., Grice, S.J., \& Karmiloff-Smith, A. (2002). Neuroimaging of typical and atypical development: a perspective from multiple levels of analysis. Development and Psychopathology, 14, 521-536.

Just, M.A., Cherkassky, V.L., Keller, T.A., Kana, R.K., \& Minshew, N.J. (2007). Functional and anatomical cortical underconnectivity in autism: evidence from an fMRI study of an executive function task and corpus callosum morphometry. Cerebral Cortex, 17, 951-961.

Just, M.A., Cherkassky, V.L., Keller, T.A., \& Minshew, N.J. (2004). Cortical activation and synchronization during sentence comprehension in highfunctioning autism: evidence of underconnectivity. Brain, 127, 1811-1821.

Kana, R.K., Keller, T.A., Cherkassky, V.L., Minshew, N.J., \& Just, M.A. (2006). Sentence comprehension in autism: thinking in pictures with decreased functional connectivity. Brain, 129, 2484-2493.

Kennedy, D.P., Redcay, E., \& Courchesne, E. (2006). Failing to deactivate: resting functional abnormalities in autism. Proceedings of the National Academy of Sciences of the United States of America, 103, 8275-8280.

Koshino, H., Carpenter, P.A., Minshew, N.J., Cherkassky, V.L., Keller, T.A., \& Just, M.A. (2005). Functional connectivity in an fMRI working memory task in high-functioning autism. NeuroImage, 24, 810-821.

Lancaster, J.L., Woldorff, M.G., Parsons, L.M., Liotti, M., Freitas, C.S., Rainey, L., Kochunov, P.V., Nickerson, D., Mikiten, S.A., \& Fox, P.T. (2000). Automated Talairach atlas labels for functional brain mapping. Human Brain Mapping, 10, 120-131. 
Lord, C., Rutter, M., \& Le Couteur, A. (1994). Autism Diagnostic Interview - Revised: a revised version of a diagnostic interview for caregivers of individuals with possible pervasive developmental disorders. Journal of Autism and Developmental Disorders, 24, 659-685.

Maas, L.C., Frederick, B.D., \& Renshaw, P.F. (1997). Decoupled automated rotational and translational registration for functional MRI time series data: the DART registration algorithm. Magnetic Resonance in Medicine, 37, $131-139$

Mann, T.A., \& Walker, P. (2003). Autism and a deficit in broadening the spread of visual attention. Journal of Child Psychology and Psychiatry, 44, $274-284$.

Milne, E., Swettenham, J., \& Campbell, R. (2005). Motion perception and autistic spectrum disorder: a review. Cahiers de Psychologie Cognitive, 23, $3-34$.

Minshew, N.J., Goldstein, G., \& Siegel, D.J. (1997). Neuropsychologic functioning in autism: profile of a complex information processing disorder. Journal of the International Neuropsycholical Society, 3, 303-316.

Mottron, L., Dawson, M., Soulières, I., Hubert, B., \& Burack, J. (2006). Enhanced perceptual functioning in autism: an update, and eight principles of autistic perception. Journal of Autism and Developmental Disorders, 36, $27-43$.

Müller, R. (2007). The study of autism as a distributed disorder. Mental Retardation and Developmental Disabilities Research Reviews, 13, 85-95.

Mundy, P. (2003). Annotation: the neural basis of social impairments in autism: the role of the dorsal medial-frontal cortex and anterior cingulate system. Journal of Child Psychology and Psychiatry, 44, 793-809.

Piven, J., Palmer, P., Jacobi, D., Childress, D., \& Arndt, S. (1997). Broader 
autism phenotype: evidence from a family history study of multipleincidence autism families. American Journal of Psychiatry, 154, 185-190.

Plaisted, K., Swettenham, J., \& Rees, L. (1999). Children with autism show local precedence in a divided attention task and global precedence in a selective attention task. Journal of Child Psychology and Psychiatry, 40, 733-742.

Serences, J.T., Shomstein, S., Leber, A.B., Golay, X., Egeth, H.E., \& Yantis, S. (2005). Coordination of voluntary and stimulus-driven attentional control in human cortex. Psychological Science, 16, 114-122.

Silk, T.J., Rinehart, N., Bradshaw, J.L., Tonge, B., Egan, G., O’Boyle, M.W., \& Cunnington, R. (2006). Visuospatial processing and the function of prefrontal-parietal networks in autism spectrum disorders: a functional MRI study. American Journal of Psychiatry, 163, 1440-1443.

Small, D.M., Gitelman, D.R., Gregory, M.D., Nobre, A.C., Parrish, T.B., \& Mesulam, M.M. (2003). The posterior cingulate and medial prefrontal cortex mediate the anticipatory allocation of spatial attention. NeuroImage, 18, 633-641.

Swisher, J.D., Halko, M.A., Merabet, L.B. McMains, S.A., \& Somers, D.C. (2007). Visual topography of human intraparietal sulcus. Journal of Neuroscience, 27, 5326-5337.

Talairach, J., \& Tournoux, P. (1988). Coplanar Stereotactic Atlas of the Human Brain. Stuttgart: Thieme.

Tardif, C., Lainé, F., Rodriguez, M., \& Gepner, B. (2007). Slowing down presentation of facial movements and vocal sounds enhances facial expression recognition and induces facial-vocal imitation in children with autism. Journal of Autism and Developmental Disorders, 37, 1469-1484.

Townsend, J., Westerfield, M., Leaver, E., Makeig, S., Jung, T., Pierce, K., 
\& Courchesne, E. (2001). Event-related brain response abnormalities in autism: evidence for impaired cerebello-frontal spatial attention networks. Cognitive Brain Research, 11, 127-145.

Townsend, J., \& Courchesne, E., (1994). Parietal damage and narrow "spotlight" spatial attention. Journal of Cognitive Neuroscience, 6, 220-232.

Townsend, J., Singer-Harris, N., \& Courchesne, E. (1996). Visual attention abnormalities in autism: delayed orienting to location. Journal of the International Neuropsychological Society, 2, 541-550.

Wang, L., Liu, X., Guise, K.G., Knight, R.T., Ghajar, J., Fan, J. Effective connectivity of the fronto-parietal network during attentional control. Journal of Cognitive Neuroscience, in press.

Wicker, B., Fonlupt, P., Hubert, B., Tardif, C., Gepner, B., \& Deruelle, C. Abnormal cerebral effective connectivity during explicit emotional processing in adults with autism spectrum disorder. Social Cognitive and Affective Neuroscience 3, 135-143.

Wojciulik, E., \& Kanwisher, N. (1999). The generality of parietal involvement in visual attention. Neuron, 23, 747-764.

Woodbury-Smith, M.R., Robinson, J., Wheelwright, S., \& Baron-Cohen, S. (2005). Screening adults for Asperger Syndrome using the AQ: a preliminary study of its diagnostic validity in clinical practice. Journal of Autism and Developmental Disorders 35, 331-335.

Zwaigenbaum, L., Bryson, S., Rogers, T., Roberts, W., Brian, J., \& Szatmari, P. (2005). Behavioral manifestations of autism in the first year of life. International Journal of Developmental Neuroscience, 23, 143-152. 


\begin{tabular}{|c|c|c|c|c|c|c|c|c|}
\hline \multirow{2}{*}{$\begin{array}{l}\text { Age } \\
\text { (Years) }\end{array}$} & \multicolumn{2}{|c|}{ WASI } & \multirow[t]{2}{*}{$\mathrm{AQ}$} & \multicolumn{3}{|c|}{ ADI-R } & \multicolumn{2}{|c|}{$d^{\prime}$} \\
\hline & VIQ & $P I Q$ & & Social & Communication & $\begin{array}{l}\text { Repetitive } \\
\text { Behaviours }\end{array}$ & Incongruent & Congruent \\
\hline \multicolumn{9}{|c|}{ Normal } \\
\hline 13.09 & 118 & 112 & 20 & & & & 1.91 & 2.21 \\
\hline 15.45 & 129 & 120 & 16 & & & & 2.16 & 2.55 \\
\hline 12.75 & 119 & 118 & 4 & & & & 1.86 & 1.65 \\
\hline 12.67 & 134 & 99 & 2 & & & & 1.12 & 1.36 \\
\hline 14.41 & 133 & 121 & 8 & & & & 0.58 & 0.91 \\
\hline 14.89 & 123 & 126 & 15 & & & & 1.60 & 2.10 \\
\hline 14.56 & 107 & 119 & 2 & & & & 1.97 & 2.06 \\
\hline 14.63 & 136 & 111 & 24 & & & & 2.57 & 1.91 \\
\hline 12.70 & 125 & 131 & 26 & & & & 1.75 & 2.08 \\
\hline 13.91 & 125 & 117 & 13.0 & & & & 1.72 & 1.87 \\
\hline 1.09 & 9 & 9 & 9.4 & & & & 0.62 & 0.49 \\
\hline
\end{tabular}

\begin{tabular}{l|ll|l|l|ll}
\hline \multicolumn{1}{l}{ Sib } \\
\hline 11.93 & 121 & 129 & 7 & 1.34 & 1.91 \\
15.81 & 121 & 103 & 7 & 0.91 & 0.29 \\
14.05 & 123 & 119 & 6 & 1.75 & 2.23 \\
11.24 & 120 & 99 & 4 & 0.67 & 1.16 \\
11.40 & & & 3 & 1.04 & 1.53 \\
11.46 & 136 & 131 & 23 & 1.58 & 2.46 \\
13.57 & & & 4 & 1.12 & 1.64 \\
\hline & & & & & 1.20 \\
12.78 & 124 & 116 & 7.7 & 0.38 & 1.60 \\
1.74 & 7 & 15 & 6.9 & &
\end{tabular}

\begin{tabular}{l|ll|llll|ll}
\hline \multicolumn{7}{c}{ ASC } \\
\hline 13.45 & 106 & 88 & 41 & 15 & 15 & 6 & 0.40 & 0.52 \\
15.55 & 126 & 93 & 43 & 26 & 17 & 8 & 1.00 & 1.46 \\
12.47 & 105 & 100 & 46 & 26 & 24 & 11 & 1.23 & 1.33 \\
11.44 & 109 & 105 & 39 & 29 & 24 & 10 & 1.01 & 1.63 \\
14.13 & 113 & 120 & 46 & 24 & 26 & 10 & 1.25 & 1.35 \\
14.68 & 88 & 93 & 32 & 20 & 14 & 4 & 1.50 & 1.68 \\
15.11 & 143 & 119 & 37 & 26 & 21 & 6 & 1.53 & 1.43 \\
10.89 & 147 & 141 & 36 & 19 & 11 & 6 & 1.81 & 1.58 \\
\hline & & & & & & & & 1.22 \\
13.47 & 117 & 107 & 40.0 & 23.1 & 19.0 & 7.6 & 0.43 & 0.37 \\
1.72 & 20 & 18 & 5.0 & 4.7 & 5.5 & 2.5 & &
\end{tabular}

Table 1

Psychometric and behavioural data. The table lists age, IQ, AQ, ADI-R scores (for the ASC group), and $d^{\prime}$ for incongruent and congruent conditions, with means and standard deviations for each group. 


\begin{tabular}{|c|c|c|c|}
\hline \multicolumn{4}{|l|}{ gion } \\
\hline $\begin{array}{ll}O F C \\
R O F C\end{array}$ & & 58 & 0 \\
\hline $\begin{array}{l}\text { LrACC } \\
\text { RrACC }\end{array}$ & $\begin{array}{r}-5 \\
5 \\
5\end{array}$ & $\begin{array}{l}14 \\
14\end{array}$ & $\begin{array}{l}19 \\
19\end{array}$ \\
\hline $\begin{array}{l}\text { RalCb } \\
\text { LamCb }\end{array}$ & $\begin{array}{r}31 \\
-14\end{array}$ & $\begin{array}{l}-53 \\
-37 \\
-37\end{array}$ & $\begin{array}{l}-41 \\
-31 \\
-31\end{array}$ \\
\hline $\begin{array}{l}\text { RamCb } \\
\text { LPyramis }\end{array}$ & $\begin{array}{r}14 \\
-16\end{array}$ & $\begin{array}{l}-37 \\
-70\end{array}$ & $\begin{array}{l}-31 \\
-20 \\
-20\end{array}$ \\
\hline $\begin{array}{l}\text { RPyramini } \\
\text { LDeclive }\end{array}$ & $\begin{array}{ll}16 \\
-4\end{array}$ & $\begin{array}{l}-70 \\
63\end{array}$ & $\begin{array}{l}-20 \\
18\end{array}$ \\
\hline $\begin{array}{l}\text { RDeclive } \\
\text { LmdThe }\end{array}$ & $\begin{array}{c}4 \\
-7\end{array}$ & $\begin{array}{l}-63 \\
-19\end{array}$ & $\begin{array}{r}18 \\
0\end{array}$ \\
\hline $\begin{array}{l}\text { Lalns } \\
\text { Ralns }\end{array}$ & $\begin{array}{r}-42 \\
42\end{array}$ & $\begin{array}{l}14 \\
14\end{array}$ & \\
\hline $\begin{array}{l}\text { RIFG } \\
\text { LMFG1 }\end{array}$ & $\begin{array}{r}-46 \\
46 \\
-25\end{array}$ & $\begin{array}{l}23 \\
23 \\
43\end{array}$ & $\begin{array}{r}7 \\
35\end{array}$ \\
\hline $\begin{array}{l}\text { LMFG2 } \\
\text { RMFG2 }\end{array}$ & $\begin{array}{r}25 \\
-33 \\
33 \\
\end{array}$ & $\begin{array}{l}45 \\
31 \\
31\end{array}$ & \\
\hline $\begin{array}{l}\text { RMFG3 } \\
\text { LMFG4 } \\
\text { RMFG4 }\end{array}$ & $\begin{array}{r}46 \\
-39 \\
39\end{array}$ & $\begin{array}{l}19 \\
22 \\
22\end{array}$ & 44 \\
\hline $\begin{array}{l}\text { RMedFG } \\
\text { LdACC }\end{array}$ & $\begin{array}{r}-1 \\
-4 \\
-4\end{array}$ & $\begin{array}{l}14 \\
-2\end{array}$ & 50 \\
\hline $\begin{array}{l}\text { LPreCG } \\
\text { RPreCG }\end{array}$ & 35 & $\begin{array}{l}-11 \\
-11\end{array}$ & 34 \\
\hline $\begin{array}{l}\text { RPhippG } \\
\text { H Hipp }\end{array}$ & $\begin{array}{l}-32 \\
-32 \\
-33\end{array}$ & $\begin{array}{l}-30 \\
-30 \\
-12\end{array}$ & $\begin{array}{l}-5 \\
-3 \\
-14\end{array}$ \\
\hline $\begin{array}{l}\text { RHipp } \\
\text { LpSTG }\end{array}$ & $\begin{array}{r}33 \\
-51 \\
-51\end{array}$ & $\begin{array}{l}-12 \\
-43\end{array}$ & 19 \\
\hline $\begin{array}{l}\mathrm{LmCu} \\
\mathrm{RmCu}\end{array}$ & $\begin{array}{l}-2 \\
-2 \\
2\end{array}$ & $\begin{array}{l}-43 \\
-80 \\
-80\end{array}$ & \\
\hline $\begin{array}{l}\text { RPCut } \\
\text { LPCu19 }\end{array}$ & $\begin{array}{r}-1 \\
-29 \\
-20\end{array}$ & $\begin{array}{l}-46 \\
-65 \\
-65\end{array}$ & $\begin{array}{l}37 \\
23 \\
22\end{array}$ \\
\hline $\begin{array}{l}\text { LiFusG } \\
\text { RIFusG }\end{array}$ & $\begin{array}{r}-40 \\
40\end{array}$ & $\begin{array}{l}-68 \\
-68 \\
-63\end{array}$ & -9 \\
\hline $\begin{array}{l}\text { RaIIIs } \\
\text { LSMG } \\
\text { PSM }\end{array}$ & $\begin{array}{r}29 \\
-40\end{array}$ & $\begin{array}{l}-53 \\
-43 \\
-42\end{array}$ & $\begin{array}{l}28 \\
2_{2}\end{array}$ \\
\hline $\begin{array}{l}\text { LAG } \\
\text { RAG }\end{array}$ & $\begin{array}{l}-37 \\
-37 \\
-51\end{array}$ & $\begin{array}{r}-66 \\
-66 \\
4\end{array}$ & $\begin{array}{r}30 \\
1\end{array}$ \\
\hline $\begin{array}{l}\text { RaSTG } \\
\text { LPCC }\end{array}$ & $\begin{array}{l}-7 \\
7\end{array}$ & $\begin{array}{r}4 \\
-24 \\
-24\end{array}$ & 20 \\
\hline $\begin{array}{l}\text { LpostCu } \\
\text { RpostCu } \\
\text { LISPL }\end{array}$ & $\begin{array}{r}-6 \\
6 \\
-25\end{array}$ & $\begin{array}{l}-82 \\
-82 \\
-60\end{array}$ & 45 \\
\hline $\begin{array}{l}\text { RISPL } \\
\text { LPSPL }\end{array}$ & $\begin{array}{r}25 \\
-13 \\
13\end{array}$ & $\begin{array}{l}-60 \\
-66\end{array}$ & 50 \\
\hline LLingG & & $\begin{array}{l}-66 \\
-73 \\
-72\end{array}$ & \\
\hline $\begin{array}{l}\text { RLingG } \\
\text { LmFusG }\end{array}$ & $\begin{array}{r}4 \\
25 \\
25\end{array}$ & $\begin{array}{l}-73 \\
-71\end{array}$ & \\
\hline $\begin{array}{l}\text { RmFusG } \\
\text { LSTG42 }\end{array}$ & & $\begin{array}{l}-71 \\
-38\end{array}$ & \\
\hline $\begin{array}{l}\text { RSTG42 } \\
\text { LmSPL }\end{array}$ & -8 & -66 & 32 \\
\hline LIPTO & $\begin{array}{r}-36 \\
36\end{array}$ & $\begin{array}{l}-60 \\
-75 \\
-75\end{array}$ & \\
\hline & 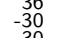 & b & \\
\hline & & 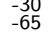 & 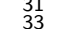 \\
\hline & & & \\
\hline
\end{tabular}

\section{Table 2}

Regions of interest for the exploratory analysis of correlation (Talairach coordinates). Abbreviations: $L$ left, $\mathrm{R}$ right, $\mathrm{r}$ rostral, $\mathrm{d}$ dorsal, a anterior, $\mathrm{p}$ posterior, I lateral, m medial, OFC orbitofrontal cortex, ACC anterior cingulate cortex, Cb cerebellum, Thal thalamus, Ins insula, IFG inferior frontal gyrus, MFG middle frontal gyrus, MedFG medial frontal gyrus, PreCG precentral gyrus, PhippG parahippocampal gyrus, Hipp hippocampal formation, STG superior temporal gyrus, Cu cuneus, PCu precuneus, FusG fusiform gyrus, IPs intraparietal sulcus, SMG supramarginal gyrus, AG angular gyrus, PCC posterior cingulate cortex, SPL superior parietal lobule, IPL inferior parietal lobule, LingG lingual gyrus, IPTO posterior intraparietal sulcus near transverse occipital sulcus. 


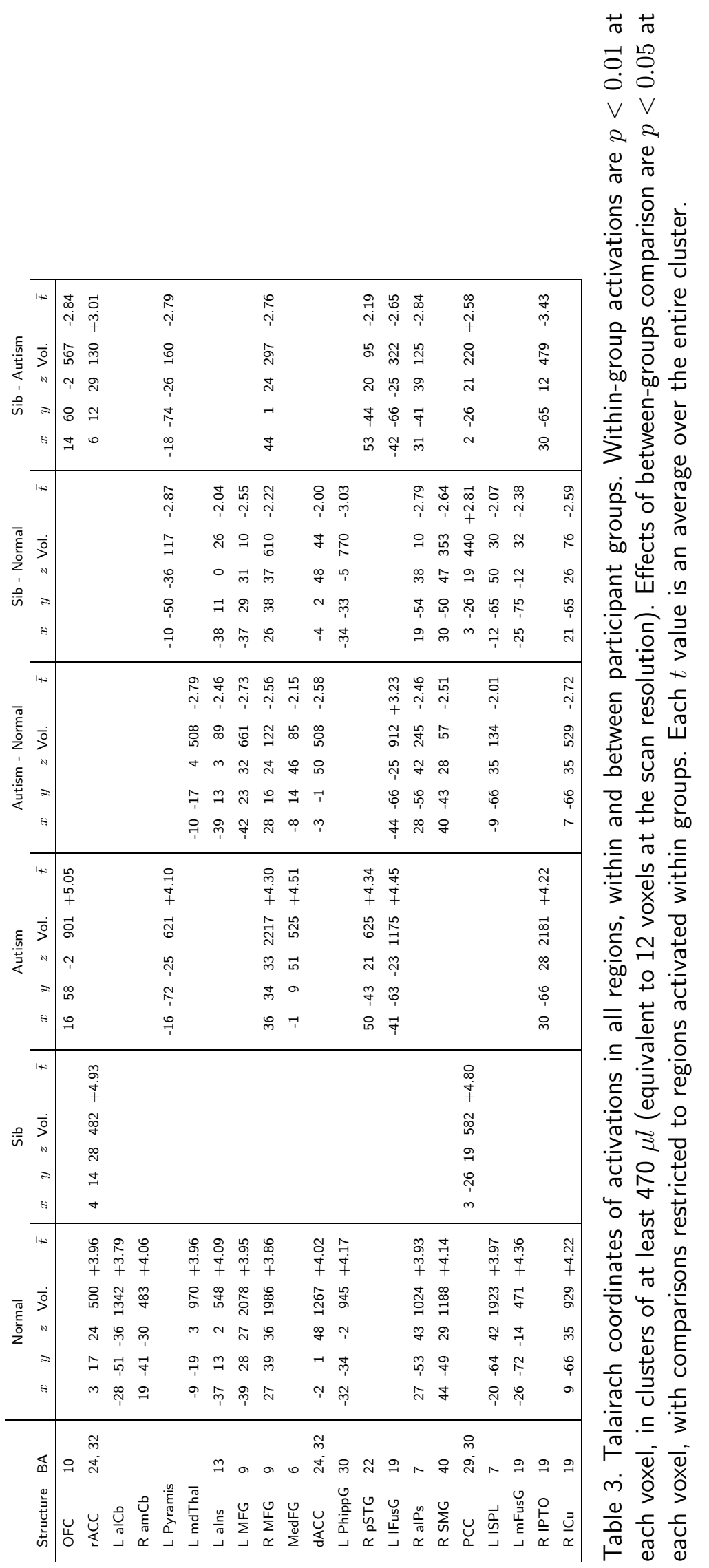


Fig. 1. Examples of incongruent (left) and congruent (right) stimuli. The middle position in each $3 \times 3$ hemifield was attended; all other positions were distractors. Each stimulus array was presented for $167 \mathrm{~ms}$. Participants were asked to report a conjunction of orientation (with ignored colour) in one target and colour (with ignored orientation) in the other.

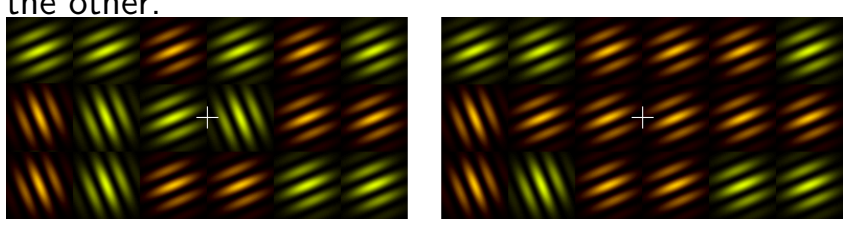




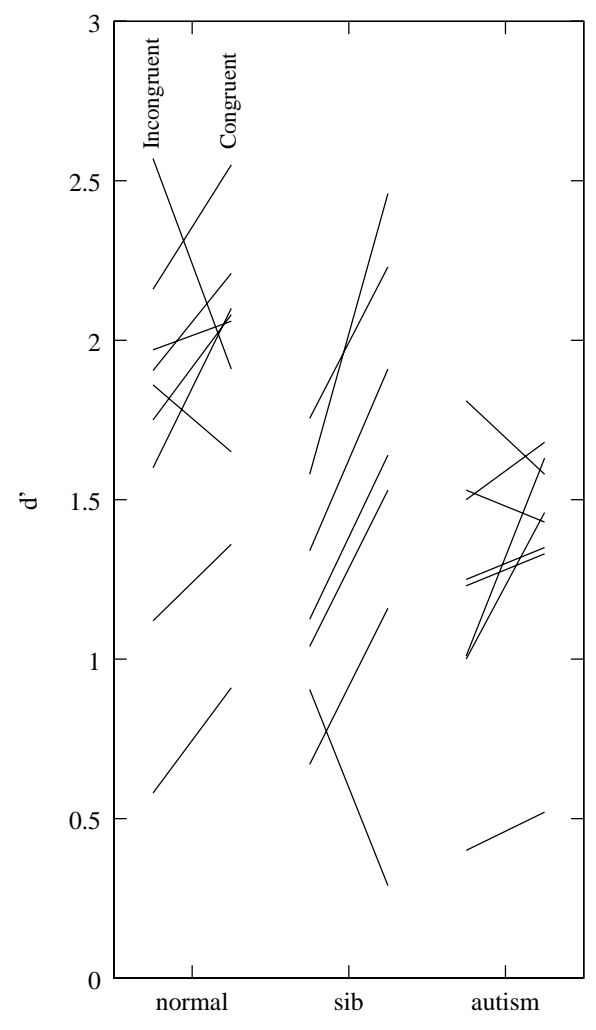

Fig. 2. $d^{\prime}$ scores as a function of diagnostic group and stimulus congruence. For each participant separately, the $d^{\prime}$ scores for incongruent stimuli and for congruent stimuli are joined by a line. Despite a large amount of variance within groups, both the sib group and the ASC group differed significantly from controls. 


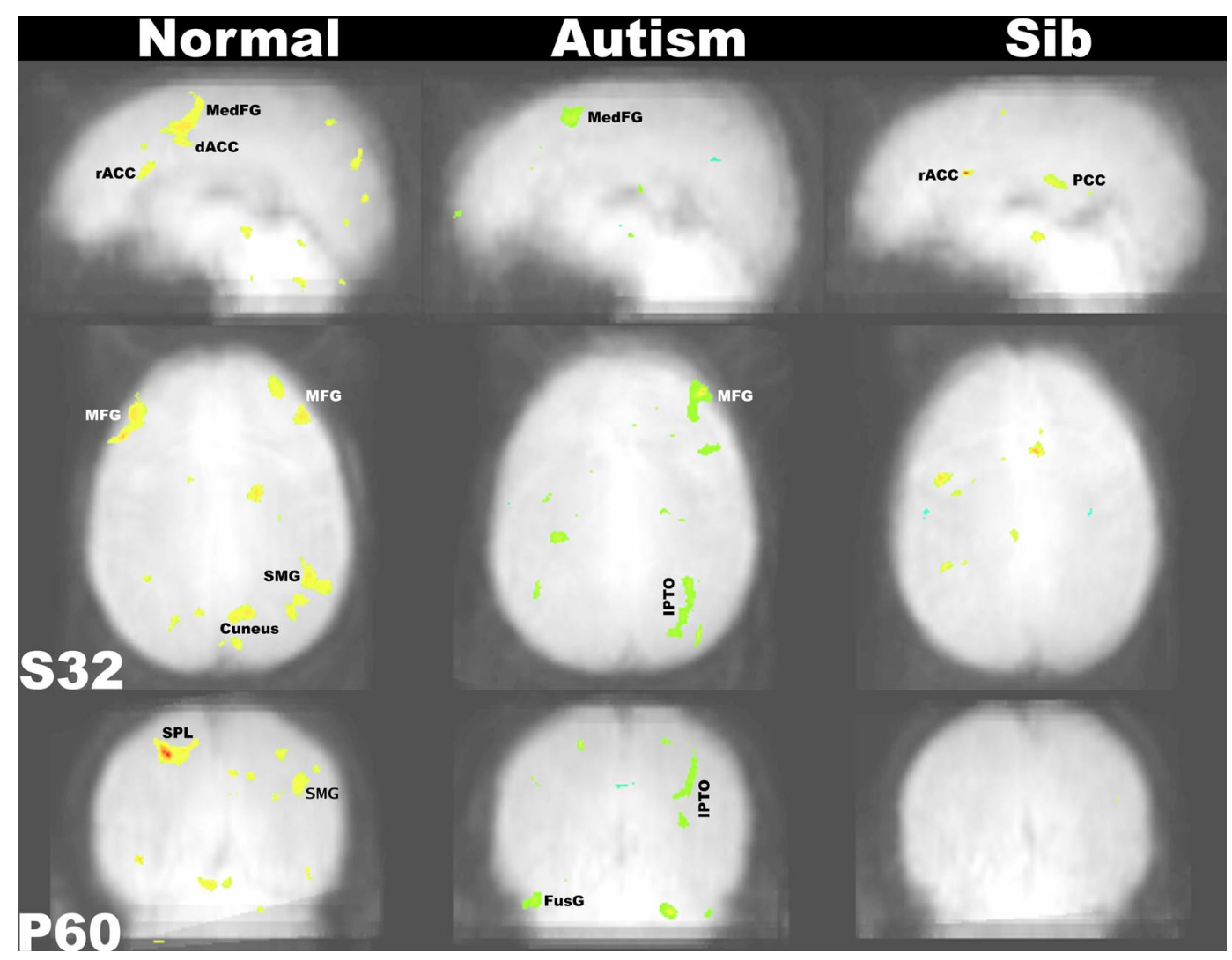

Fig. 3. Activation images in within-group averaged echo-planar slices, illustrating group differences in patterns of activation. See Table 3 for contrasts. Left and right are true left and right. 

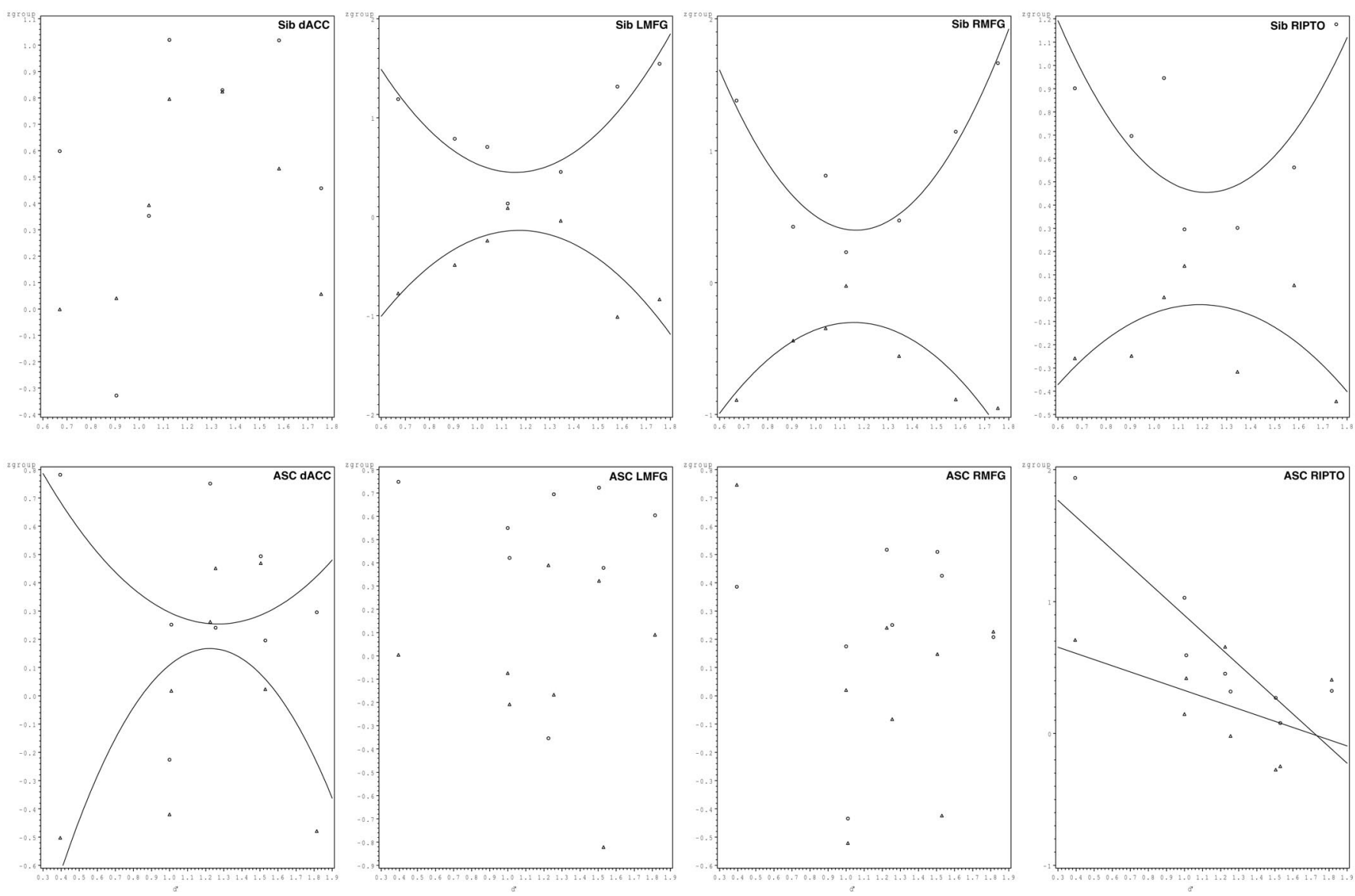

Fig. 4. Relationship of behavioural accuracy to differences in fMRI time course. Each participant has one datum on the abscissa $\left(d^{\prime}\right)$ and two on the ordinate (Fisher's $z$-transforms of correlations with the participant's own group's average fMRI time series (circles) and with the control group's (triangles)). Regression curves are illustrated where significant relationships between $d^{\prime}$ and $z_{\text {group }}-z_{\text {normal }}$ have been detected. 

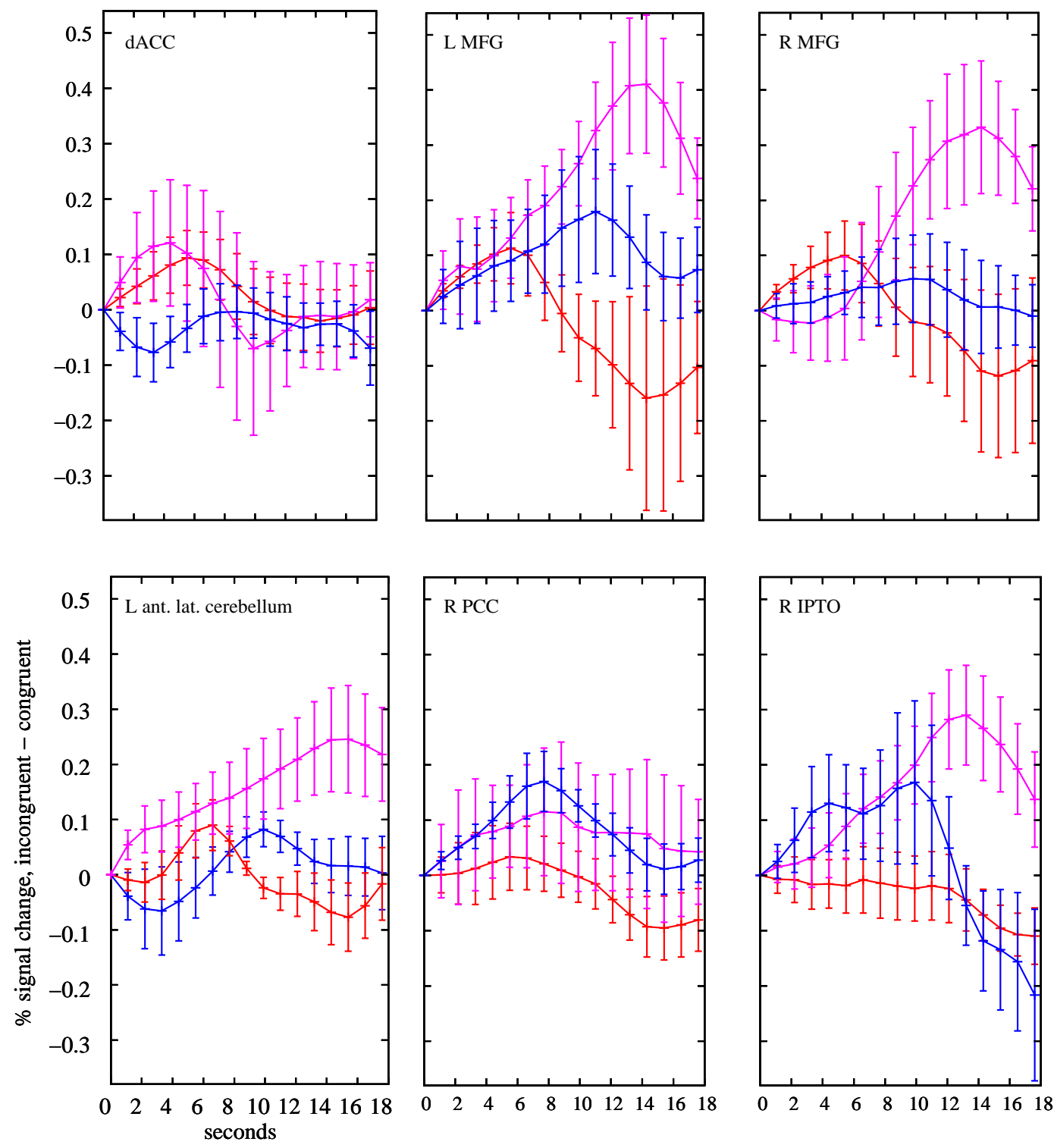

Fig. 5. fMRI time series for regions in which time series examinations revealed activation characteristics not captured by the hæmodynamic model. Dorsal anterior cingulate activation in sibs was highly variable across individuals. Delayed activations in ASC and sibs arose in middle frontal gyrus, the cerebellar attention area, and posterior intraparietal sulcus. ASC activation of posterior cingulum, though subthreshold, was closer to that of sibs than controls. Time courses are plotted for controls (red), ASC (blue), and sibs (magenta). Bars represent standard error of the mean. 

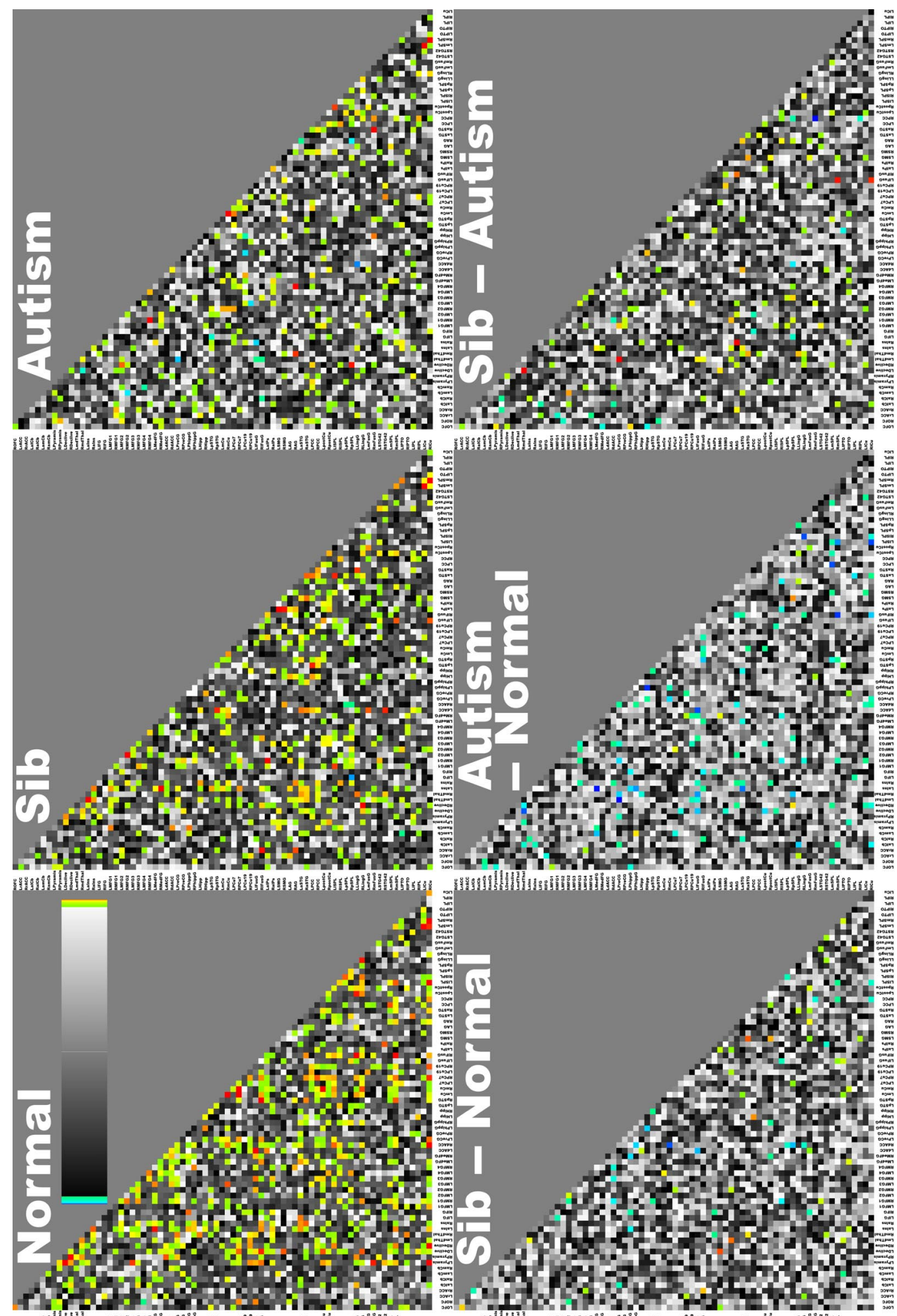

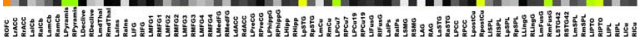

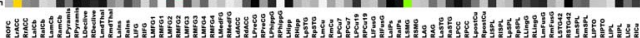

Fig. 6. Correlations of event-related response amongst 38 bilateral pairs of brain regions. Within-group and between-groups comparisons significant at two-tailed $\alpha=0.05$ are denoted in colour (see the scale bar at top left). The comparison between ASC and the other two groups was statistically significant when data were pooled across regions, as reflected in the overall weakness of correlations in the ASC group (top right panel) versus strength in the control and sib groups (top left two panels). Group comparisons within regions, however, have not been corrected for multiple comparisons and therefore are considered exploratory. 